\title{
Interference mitigation: impact on GNSS timing
}

\author{
Daniele Borio $^{1}\left[{ }^{[} \cdot\right.$ Ciro Gioia $^{1}[$
}

Received: 31 July 2020 / Accepted: 17 December 2020 / Published online: 19 January 2021

(C) The Author(s) 2021

\begin{abstract}
While interference mitigation techniques can significantly improve the performance of a Global Navigation Satellite System (GNSS) receiver in the presence of jamming, they can also introduce distortions, biases and delays on the GNSS measurements and on the final receiver solution. We analyze the impact of five interference mitigation techniques on the solution provided by a GNSS timing receiver that operates in a known location and under static conditions. In this configuration, the receiver only estimates its clock bias and drift, which can be potentially affected by interference mitigation. The analysis has been performed considering a multiconstellation case, including GPS L1 Coarse Acquisition (C/A), Galileo E1b/c and Beidou B1c signals. Tests were also conducted on the wideband Galileo E5b modulation. In all cases, real jammers were used to challenge GNSS signal reception. The techniques analyzed are four Robust Interference Mitigation (RIM) approaches and the Adaptive Notch Filter (ANF). From the analysis, it emerges that RIM techniques do not affect the receiver clock bias and drift. On the other hand, the ANF introduces a modulation-dependent delay on the clock bias. This delay is difficult to predict and is common to signals adopting modulations with similar spectral characteristics. In this respect, interoperable signals such as the Galileo E1b/c and Beidou B1c components are affected in the same way by the ANF, which leaves the Galileo-Beidou intersystem bias unaltered. Stability analysis has also been performed: interference mitigation does not significantly increase the short-term characteristics of the estimated clock bias and drift for low jamming levels.
\end{abstract}

Keywords Adaptive notch filter $\cdot$ Pulse blanking $\cdot$ GNSS $\cdot$ Interference mitigation $\cdot$ Jamming $\cdot$ Timing solution

\section{Introduction}

Interference mitigation techniques can significantly improve Global Navigation Satellite System (GNSS) receiver performance in the presence of jamming and of other unwanted Radio-Frequency (RF) emissions. An overview of the RF threat can be found in the book edited by Dovis (2015) and in the review paper (Gao et al. 2016). As discussed by Dovis (2015), most interference mitigation techniques are implemented at the early stages of the receiver and operate on the digital samples provided by the receiver front end. In this way, significant performance improvements can be achieved, mostly at the signal processing stages such as acquisition and tracking. While interference mitigation can enable receiver processing in the presence of significant levels of jamming, distortions, biases and delays can be introduced in the GNSS

Daniele Borio

daniele.borio@ec.europa.eu

1 Joint Research Centre, European Commission, Via Enrico Fermi 2749, 21027 Ispra (VA), Italy measurements and in the final Position, Velocity, and Time (PVT) solution. Distortions at the measurement and position level were analyzed in our study (Borio and Gioia 2020a) for five interference mitigation techniques. Raasakka and Orejas (2014) and Di Grazia et al. (2019) studied the impact of Adaptive Notch Filters (ANFs) on different receiver stages showing that this technique introduces biases on the GNSS measurements. These studies, however, did not consider the impact of interference mitigation on timing solutions.

In this work, we experimentally evaluate the impact of interference mitigation on the solution computed by a timing receiver using real data collected in the presence of jamming. In a timing receiver, the user position is assumed known and the receiver is operated in static conditions as discussed in Guo et al. (2019). In this way, the receiver needs to estimate only its clock bias and drift, which are then used to steer the receiver clock to a GNSS time scale or Universal Time Coordinated (UTC). The five interference mitigation techniques considered in this work are four Robust Interference Mitigation (RIM) approaches and the ANF. The RIM framework was introduced by Borio (2017) and further 
developed by Borio and Closas (2018). Moreover, Borio and Gioia (2020a) assessed the impact of RIM in the measurement and position domains. The use of ANF for GNSS interference mitigation was suggested by Calmettes et al. (2001). A single-pole version of the ANF was proposed by Borio et al. (2006), whereas Qin et al. (2019a) analyzed its performance when tracking fast-varying swept interference. RIM is a class of mitigation techniques operating at the signal processing level and using principles of robust statistics. In particular, the concept of M-estimators as introduced by Huber (1964) is used to define RIM. A review of statistical robustness can be found in Huber and Ronchetti (2009). Pulse Blanking (PB) (Gao et al. 2013; Bastide et al. 2004) and frequency-domain excision (Young and Lehnert 1998) belong to the class of RIM approaches. ANF is a form of Interference Cancelation (IC) and is effective in reducing the impact of Continuous Wave (CW) and swept interference as reviewed by Dovis (2015) and studied by Qin et al. (2019a). These techniques are briefly reviewed in the following.

In this study, we assessed the impact of the above-mentioned techniques on a GNSS timing solution. The analysis has been performed considering a multiconstellation configuration, including GPS L1 Coarse Acquisition (C/A), Galileo E1b/c and Beidou B1c signals. We also conducted tests on the wideband Galileo E5b modulation. In all cases, real jammers were used to challenge GNSS signal reception and specific focus was devoted to the impact on the clock bias and drift estimated by the receiver. In this respect, the analysis performed complements the results we provided in Borio and Gioia (2020a), where the impact of five interference mitigation techniques was analyzed in the measurement and position domains. In our previous study, the impact on timing parameters was not analyzed and the traditional PVT approach was used. In addition, we have extended the analysis to Beidou signals that were not considered before.

Two main points are investigated: (1) the potential introduction of biases in the receiver clock bias, clock drift and intersystem biases and (2) the potential increase in the Allan Deviation (ADEV) of clock time series. Reviews on the ADEV and its applications can be found in Riley (2008) and Bregni (2002). The introduction of biases has been studied by comparing the clock time series obtained with and without interference mitigation. The time series obtained without interference mitigation has been used as a reference, and differences were formed using the time series computed using interference mitigation. The analysis shows that clock bias differences are zero mean and RIM introduces no biases. On the contrary, a time-varying delay is introduced by the ANF. This delay depends on the ANF parameters and on the spectral characteristic of the GNSS signals considered. In this respect, the GPS L1 C/A signal experiences larger delays than the Galileo and Beidou modulations in the tests performed. Galileo E1b/c and Beidou B1c signals are interoperable and are characterized by the same spectral characteristics. For this reason, the same delay is introduced by the ANF on these signals. The fact is also supported by the analysis performed on the intersystem biases, which are affected by the ANF only when the involved GNSSs adopt signals with different spectral characteristics. In all cases, no biases were found in the clock drifts.

The potential increase of ADEV was analyzed using the approach discussed by Gioia and Borio (2016) and considering low jamming conditions. The aim was to assess potential losses of efficiencies caused by interference mitigation. In particular, the stability of the clock solution has been computed using clock bias and drift time series. The ADEV curves obtained under different conditions are practically coincident with differences only for reduced averaging time intervals. This shows the limited impact of interference mitigation on the short-term stability of the receiver clock time series.

The analysis has been performed using a Software Defined Radio (SDR) approach, processing the same datasets used in Borio and Gioia (2020a) for the position-domain analysis. The approach adopted for processing the collected signals is detailed in the section on timing solution and timing metrics.

This is an extended version of Borio and Gioia (2020b). Conference work was significantly extended by analyzing the intersystem biases and the clock drifts. Moreover, in our previous work, Beidou signals were not considered and only RIM techniques were analyzed. The analysis of Beidou B1c signals and the impact of the ANF are new contributions of this work.

The next section briefly reviews the five interference mitigation techniques considered. The processing strategy adopted to investigate the impact of interference mitigation on the timing solution is then introduced along with the timing metrics adopted for the analysis. The experimental setup and the test results are then discussed. Finally, conclusions are provided.

\section{Interference mitigation techniques}

In a GNSS receiver, the analog GNSS signals are collected by the antenna and converted to baseband by the receiver front end as reviewed in Tsui (2004) and Kaplan and Hegarty (2005). The final output of the front end is a sequence of digital samples, $y[n]$, that are used by the subsequent receiver stages to generate measurements and compute the receiver position and clock parameters. A schematic representation of the different receiver blocks is provided in Fig. 1. When RF interference is present, the digital samples, $y[n]$, will also be contaminated by an interference term, $i[n]$. In order to mitigate its impact, digital signal processing techniques can be adopted as reviewed in Dovis (2015). In Fig. 1, the block 


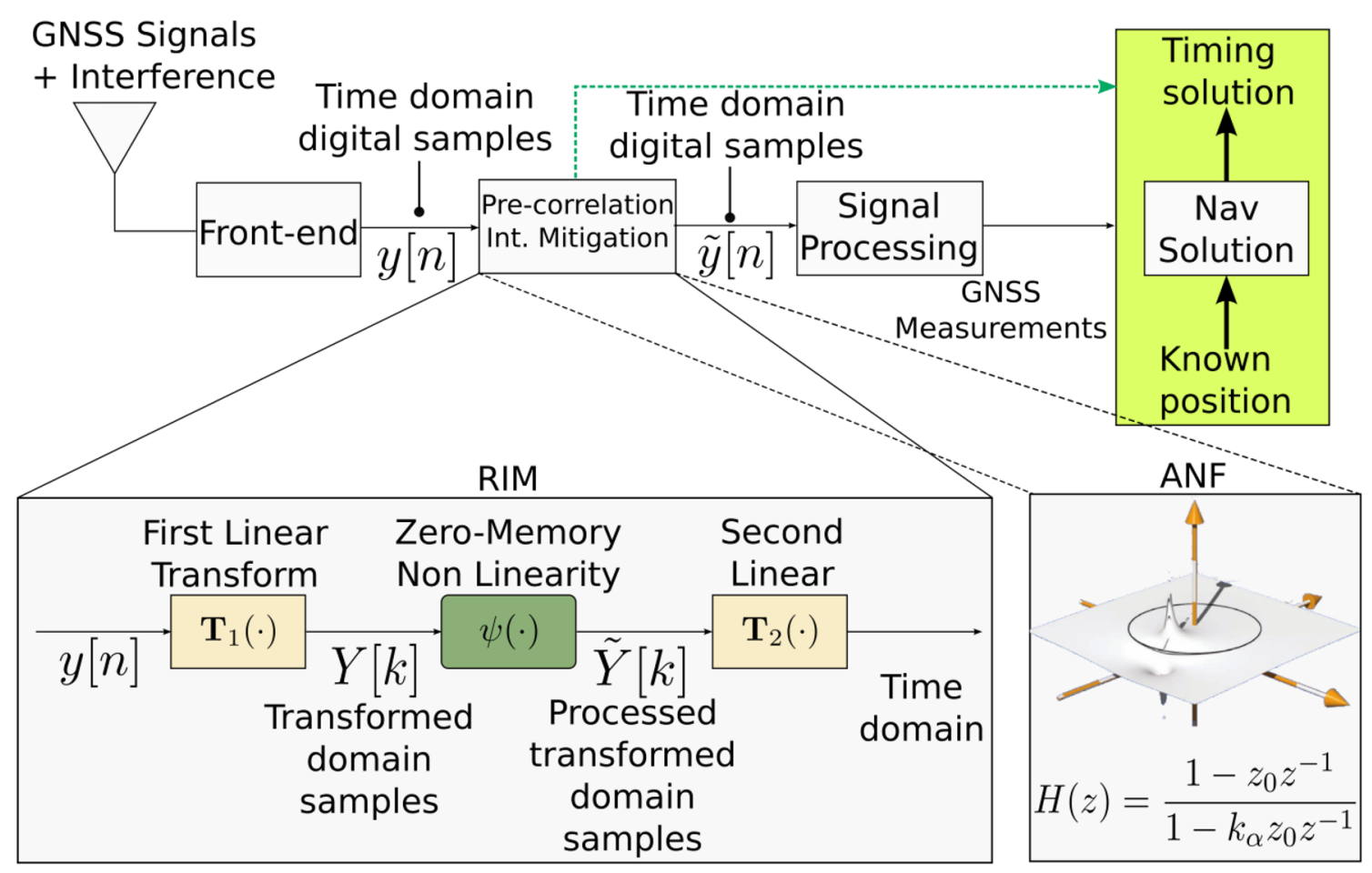

Fig. 1 Schematic representation of the processing scheme adopted to test the impact of interference mitigation techniques on a GNSS timing solution

denoted as 'pre-correlation interference mitigation' implements such algorithms and aims at mitigating the impact of interference operating directly on the input samples. This block produces a new sequence of digital samples, $y[n]$, that are used for acquisition, tracking, measurement generation and navigation solution computation.

Interference mitigation techniques applied at the sample level can introduce distortions on the measurements and the position solution as shown in Borio and Gioia (2020a). In this work, we analyze the impact of five interference mitigation techniques on the timing solution generated by a multiconstellation GNSS timing receiver (Guo et al. 2019; Xiangwei et al. 2015). In this respect, the user position is considered known and only the clock bias and clock drifts are computed by the receiver. If no receiver calibration is performed, the intersystem biases between the different constellations need to be estimated. Figure 1 also illustrates the two classes of interference mitigation techniques considered in this study: RIM and the ANF.

The five interference mitigation techniques analyzed can be classified according to the schematic representation provided in Fig. 2. Interference mitigation techniques can be designed according to the IC principle (Madhani et al. 2003): the mitigation algorithm estimates at first the interfering signal that is then removed (canceled) from the input samples. The ANF uses this principle and estimates the parameters of frequency-modulated signals. IC mitigation techniques usually adopt a signal model for the interfering signal. The ANF assumes that the interfering signal has a practically constant amplitude and that it can be estimated by the past samples (Borio et al. 2006, 2008). In particular, the ANF is characterized by the following transfer function:

$H(z)=\frac{1-z_{0} z^{-1}}{1-k_{\alpha} z_{0} z^{-1}}$

where $k_{\alpha}$ is the pole contraction factor and $z_{0}$ is the notch zero, which is continuously estimated by the notch adaptation block. The complete description of the ANF and the analysis of the criteria behind the selection of its parameters are outside the scope of this work. In the following, two values for the pole contraction factor $k_{\alpha}=0.8$ and 0.9 are used. These values correspond to the optimal parameter settings derived in (Qin et al. 2019a, 2019b). The ANF places a narrow notch around the frequency

$f_{0}=\frac{f_{s}}{2 \pi} \angle z_{0}$

where $f_{s}$ is the sampling frequency used to sample the input analog signal and produce the samples, $y[n]$. Signal components around $f_{0}$ are removed by the filter notch that should track the interference term. Alternatively to the IC, mitigation techniques can be designed using principles from robust statistics (Huber and Ronchetti 2009; Borio 2017). 
Fig. 2 Schematic representation of the five interference mitigation techniques analyzed and their relationships

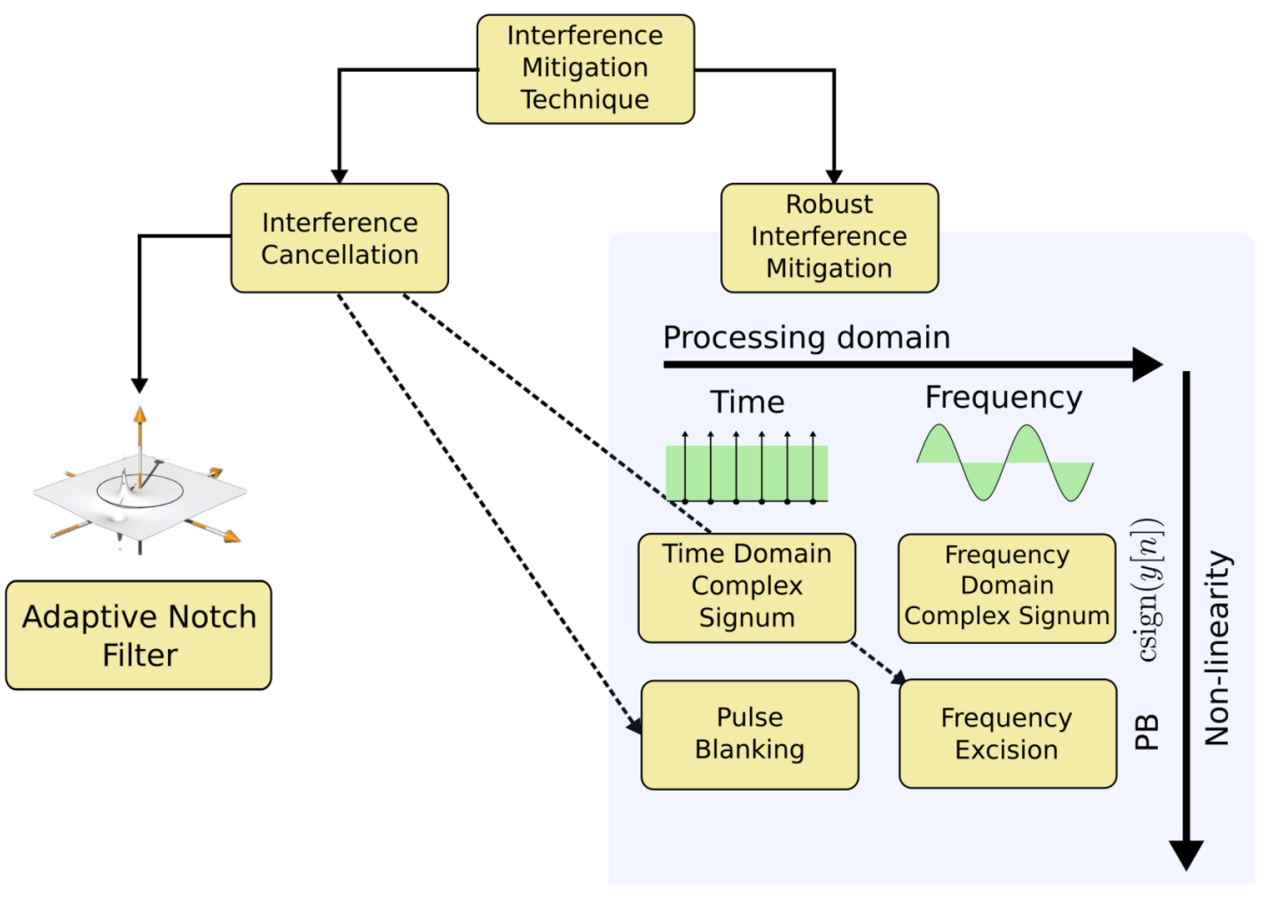

In this case, the interfering signal is first projected into a transformed domain where it is expected to assume a sparse representation, as discussed in Borio and Closas (2019). In the sparse interference domain, only a limited number of samples are affected by interference and can be treated as outliers. In particular, the transform-domain samples are computed as

$Y[k]=\boldsymbol{T}_{1}(y[n])$

where $\boldsymbol{T}_{\boldsymbol{l}}(\bullet)$ defines the transformation bringing $N$ timedomain samples into a transform domain where interference admits a sparse representation. In the following, only time and frequency domains are considered. Time-domain processing is obtained by considering as $\boldsymbol{T}_{\boldsymbol{I}}(\bullet)$, the identity, i.e., no transform is applied to the input samples. Frequencydomain approaches are obtained using the Discrete Fourier Transform (DFT)/Fast Fourier Transform (FFT).

A nonlinearity is then used to mitigate the impact of these outliers. PB and frequency excision are obtained when the following nonlinearity

$\tilde{Y}[k]=\psi(Y[k])=\left\{\begin{array}{c}Y[k] \text { if }|Y[k]|<T_{h} \\ 0 \quad \text { otherwise }\end{array}\right.$

is adopted. In (4), $T_{h}$ is a decision threshold fixed by the user. In the following $T_{h}=3 \sigma$ has been adopted where $\sigma^{2}$ is the variance of the noise affecting $Y[k]$ and estimated in the absence of interference. When processing is performed directly in the time domain, PB is obtained. If a DFT is used to bring the samples in the frequency domain, then frequency excision is implemented. Incidentally, PB and frequency excision can also be interpreted as forms of IC where in the first case, the interference term is modeled as a sequence of pulses and in the second as a combination of complex sinusoids.

\section{Nonlinearity}

$\tilde{Y}[k]=\operatorname{csign}(Y[k])=\frac{Y[k]}{|Y[k]|}$

defines the complex signum RIM that does not require any parameter setting (Borio and Closas 2018). Finally, a second transform is applied to bring back the samples in the time domain. For time-domain processing, also the second transform is equal to the identity. For frequency-domain processing, the Inverse DFT (IDFT)/Inverse IFFT (IFFT) is used:

$\tilde{y}[n]=\boldsymbol{T}_{2}(\tilde{Y}[k])$

The combination of two processing domains and two nonlinearities leads to the four RIM approaches analyzed:

- Time-Domain Complex Signum (TDCS),

- Time-Domain Pulse Blanking (TDPB),

- Frequency-Domain Complex Signum (FDCS),

- Frequency-Domain Pulse Blanking (FDPB).

These are the same techniques considered in our previous study for the measurement and position-domain analysis (Borio and Gioia 2020a). 


\section{Timing solution and timing metrics}

The samples generated after interference mitigation, $\tilde{y}[n]$, have been processed using a custom MATLAB software receiver supporting multiple constellations and several GNSS frequencies. The software developed has been used to process:

- signals from the L1 frequency (1575.42 MHz), including the GPS L1 C/A modulation (Kaplan and Hegarty 2005), the Galileo E1b/c signal (European Union 2016) and the Beidou B1c component (Mingquan et al. 2019),

- the wideband Galileo E5b component (European Union 2016).

The Galileo E1b/c and the Beidou B1c signals adopt a Composite Binary Offset Carrier (BOC) (CBOC) (Hein et al. 2006) and a Quadrature Multiplexed BOC (QMBOC) (Mingquan et al. 2019), respectively. These modulations are obtained by combining a $\operatorname{BOC}(1,1)$ component with a $\operatorname{BOC}(6,1)$ modulation. The $\operatorname{BOC}(6,1)$ component has been ignored for the processing, and the two signals have been treated as $\operatorname{BOC}(1,1)$. Moreover, both Galileo E1b/c and the Beidou B1c signals have a data/pilot structure. Pilot processing has been implemented, and a pure Phase Lock Loop (PLL) has been adopted to improve performance (Kaplan and Hegarty 2005). The Galileo E5b signal is characterized by a Binary Phase Shift Keying (BPSK) (10) modulation with a data/pilot structure. Also in this case, pilot processing has been implemented. The software receiver has been used to generate Receiver Independent Exchange Format (RINEX) files used for the computation of the timing solution as better described in the next section.

\section{Timing solution algorithm}

The algorithm adopted for computing the timing solution is based on the traditional approach used by timing receivers and discussed by Xiangwei et al. (2015), where the receiver position is considered known and the receiver coordinates and velocity components are not included in the navigation solution estimation. Additional details on the algorithm adopted can be found in Gioia and Borio (2016). In particular, the antenna position was carefully presurveyed, the receiver was kept static and the velocity unknowns were set to zero. In this way, only the clock biases and drifts were estimated. In the multiconstellation case, GPS, Galileo and Beidou measurements have been used to estimate the clock biases/drifts with respect to their relative time scale. Since the receiver position and velocity are known, estimating clock biases/drifts with respect to the different GNSS time scales leads to a separable solution where measurements from the different systems contribute only to the related clock bias and drift.

Clock biases were computed using pseudorange measurements, whereas clock drifts were obtained from pseudorange rate/Doppler observations. Pseudoranges and Doppler shifts were corrected for the satellite clock errors, relativistic effect and Sagnac effect. Ionospheric errors were reduced using the Klobuchar algorithm, while the tropospheric errors were corrected using the Saastamoinen model (Kaplan and Hegarty 2005; Parkinson et al. 1996). The estimation technique used was a Weighted Least Squares (WLS) with weights fixed according to the satellite elevation (Kuusniemi 2005).

The full processing chain adopted for the analysis is shown Fig. 3. Pseudoranges and pseudorange rates were generated by the MATLAB software receiver using the different interference mitigation techniques discussed in the previous section. The figure also indicates the three tests performed and better described in the section detailing the experimental setup.

Measurements were used for the computation of the clock biases and drifts with respect to the different GNSS time scales. In the following, clock biases and drifts are indicated as $b_{s i g}[k]$ and $d_{s i g}[k]$, respectively. Subscript 'sig' is used to denote the different signals used for the clock bias/drift computation, and $k$ is the epoch index. Measurements and clock time series have been generated at a rate equal to $1 \mathrm{~Hz}$, and $k$ denotes the different 1 -s epochs.

In the multiconstellation solution, intersystem biases and drifts were also analyzed. In particular, intersystem biases were computed as

$i b_{\mathrm{GPS}-\mathrm{Gal}}[\mathrm{k}]=b_{\mathrm{L} 1 \mathrm{C} / \mathrm{A}}[\mathrm{k}]-\mathrm{b}_{\mathrm{E} 1 \mathrm{C}}[\mathrm{k}]$

$i b_{\mathrm{GPS}-\mathrm{BDS}}[\mathrm{k}]=b_{\mathrm{L} 1 \mathrm{C} / \mathrm{A}}[\mathrm{k}]-\mathrm{b}_{\mathrm{B} 1 \mathrm{C}}[\mathrm{k}]$

$i b_{\mathrm{Gal}-\mathrm{BDS}}[\mathrm{k}]=b_{\mathrm{E} 1 \mathrm{C}}[\mathrm{k}]-\mathrm{b}_{\mathrm{B} 1 \mathrm{C}}[\mathrm{k}]$

The intersystem biases (7) do not correspond to the actual Galileo/GPS Time Offset (GGTO), BDS/GPS Time Offset (BGTO) and Galileo-to-Beidou time offsets, but are the intersystem biases as estimated by the receiver and include hardware and software delays as introduced by the front end and by the processing schemes used to determine the timing solution.

Similarly, intersystem drifts were computed as

$i d_{\mathrm{GPS}-\mathrm{Gal}}[\mathrm{k}]=d_{\mathrm{L} 1 \mathrm{C} / \mathrm{A}}[\mathrm{k}]-\mathrm{d}_{\mathrm{E} 1 \mathrm{C}}[\mathrm{k}]$

$i d_{\mathrm{GPS}-\mathrm{BDS}}[\mathrm{k}]=d_{\mathrm{L} 1 \mathrm{C} / \mathrm{A}}[\mathrm{k}]-\mathrm{d}_{\mathrm{B} 1 \mathrm{C}}[\mathrm{k}]$

$i d_{\mathrm{Gal}-\mathrm{BDS}}[\mathrm{k}]=d_{\mathrm{E} 1 \mathrm{C}}[\mathrm{k}]-\mathrm{d}_{\mathrm{B} 1 \mathrm{C}}[\mathrm{k}]$ 


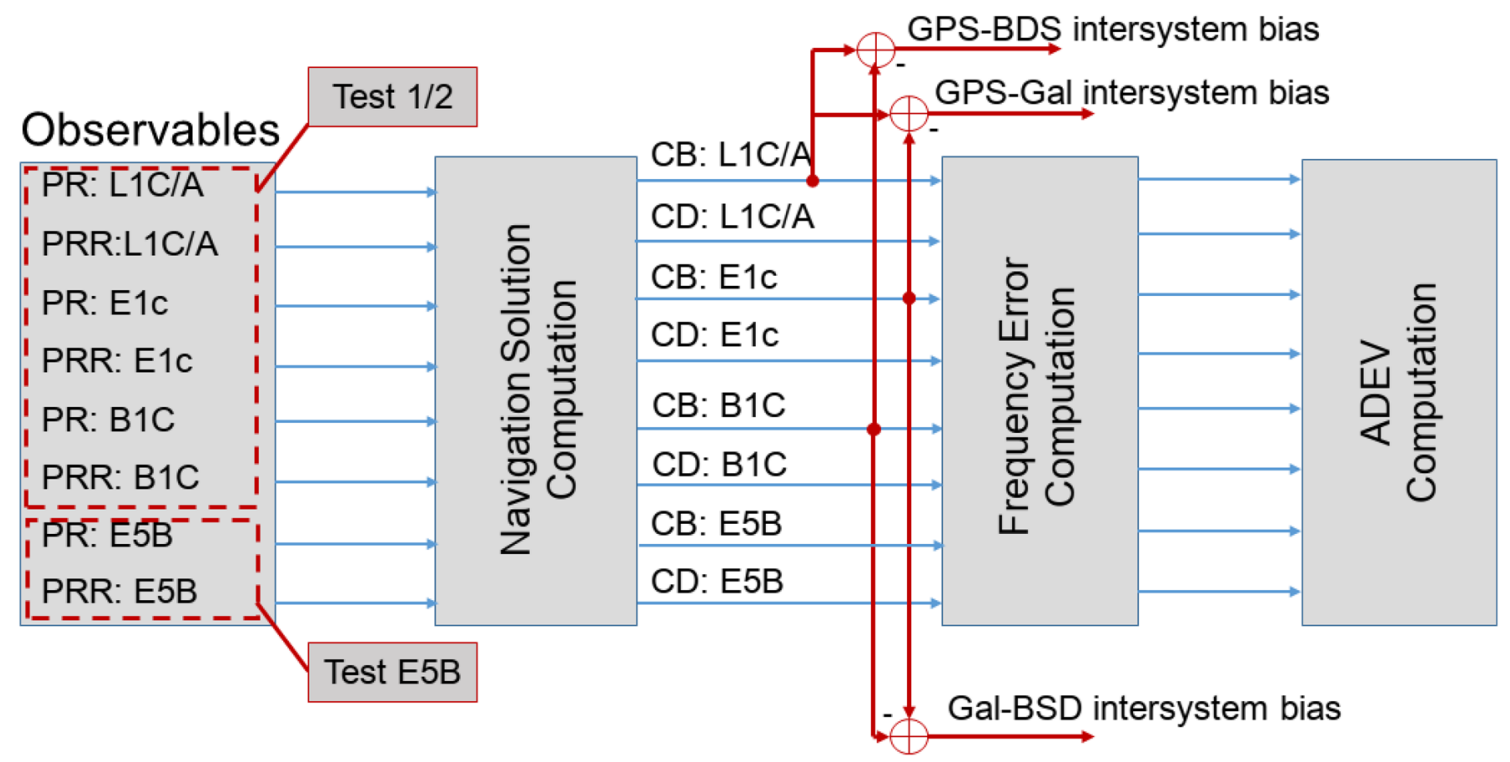

Fig. 3 Diagram of the processing chain adopted in this study. Pseudorange (PR) and pseudorange rate (PRR) are generated by a custom MATLAB software receiver and using the interference mitigation

The clock bias and drift time series were then finally used to compute frequency errors and perform stability analysis, as discussed in the next section.

\section{Stability analysis}

The ADEV is a metric widely used to characterize the stability of random processes with stationary increments, providing indications about the expected frequency deviation that can occur in a specific time interval (Riley 2008; Bregni 2002). It is used here to investigate potential degradations, mainly in the short term, introduced by interference mitigation techniques.

The variations of the timing signal generated by a clock can be captured by the normalized frequency error time series, which is then used for the computation of the ADEV. Normalized frequency error time series can be computed either from the clock bias or from the clock drift. In particular, given an averaging interval

$\tau=K T_{r}$

where $K$ is an integer and $T_{r}=1 \mathrm{~s}$ is the sampling interval of the clock bias and drift, clock bias derived normalized frequency errors can be computed as (Gioia and Borio 2016):

$\Delta_{b}[k]=\frac{b[k]-b[k-K]}{K T_{r}}$ techniques discussed in the section on interference mitigation techniques. In the figure, $\mathrm{CB}$ stands for clock bias and CD clock drift

where the clock bias, $b[k]$, is expressed in seconds and the subscript 'sig' has been omitted for ease of notation. When the clock drift is used, normalized frequency errors are computed as:

$\Delta_{d}[k]=\frac{1}{K} \sum_{i=0}^{K-1} d[k-i]$

where $d[k]$ is expressed in seconds/second and the subscript 'sig' has been dropped for the ease of notation.

Finally, normalized frequency errors are used for the computation of the ADEV, as discussed by Riley (2008):

$\operatorname{ADEV}(\tau)=\sqrt{\frac{1}{2\left(N_{k}-1\right)} \sum_{i=1}^{N_{k}-1}(\Delta[i K]-\Delta[(i-1) K])^{2}}$

where the subscript, ' $b$ ' or ' $d$ ', has been removed from the normalized frequency errors to indicate that the ADEV can be computed either from clock biases or from clock drifts. $N_{k}$ is the number of normalized frequency errors available for an averaging interval, $\tau=K T_{r}$. Additional details for the computation of the ADEV from GNSS measurements can be found in Gioia and Borio (2016).

The ADEV quantifies the expected variations of a time series over the time interval specified by $\tau$. While (12) provides the baseline approach for stability analysis, more efficient estimators using all the available data can 
be adopted (Riley 2008). In this respect, the Overlapping ADEV (OADEV) can be used:

$\operatorname{OADEV}(\tau)=\sqrt{\frac{1}{2\left(N_{1}-K\right)} \sum_{i=K}^{N_{1}-1}(\Delta[i]-\Delta[i-K])^{2}}$

where $N_{1}$ is the number of normalized frequency errors available for $K=1$. In the following, the OADEV is used for the analysis.

\section{Experimental setup}

The analysis has been performed using the same experimental setup and the same data used in Borio and Gioia (2020a) for the measurement/position-domain analysis. For this reason, only limited details are provided on the experimental setup. Data were collected using a Universal Software Radio Peripheral (USRP) II platform and processed using a customized MATLAB software receiver. The MATLAB tool has been adopted for the measurement generation and for the evaluation of the clock parameters (bias and drift). A jammer was placed inside a shielding box whose output was connected to a variable attenuator for the tests. The signal at the output of the variable attenuator was connected through a signal combiner to the RF input of the USRP. The other end of the signal combiner was connected to a roof-top antenna used to collect clean GNSS signals. In this way, it was possible to mix clean GNSS signals with a jamming component with a variable power level. The power levels were determined by the configuration adopted for the variable attenuator. In the following, three tests are considered:

Test 1: performed on the GPS/Galileo/Beidou L1 frequency band (1575.42 MHz) with an eight-bit quantization. This test was used to analyze the impact of RIM on the GPS BPSK(1) modulation and on the Galileo/Beidou $\operatorname{BOC}(1,1)$ component.

Test 2: $\quad$ also performed on the GPS/Galileo/Beidou L1 frequency band but with a 16-bit quantization. The number of bits used for quantization plays a significant role in the receiver performance in the presence of interference. This test was conducted to analyze potential differences between 8-bit/16-bit quantization.

Test E5b: performed on the Galileo E5b frequency band (1207.14 MHz). This test was conducted to analyze the impact of mitigation techniques on a wideband GNSS modulation.
Table 1 Parameters used for the different tests

\begin{tabular}{llll}
\hline Parameter & $\begin{array}{l}\text { Test } 1 \\
\text { Value }\end{array}$ & $\begin{array}{l}\text { Test } 2 \\
\text { Value }\end{array}$ & $\begin{array}{l}\text { Test E5B } \\
\text { Value }\end{array}$ \\
\hline Sampling frequency & $10 \mathrm{MHz}$ & $10 \mathrm{MHz}$ & $25 \mathrm{MHz}$ \\
Center frequency & $1575.42 \mathrm{MHz}$ & $1575.42 \mathrm{MHz}$ & $1207.14 \mathrm{MHz}$ \\
Sampling type & Complex, IQ & Complex, IQ & Complex, IQ \\
No. bits & 8 & 16 & 8 \\
Attenuation step & $1 \mathrm{~dB}$ & $1 \mathrm{~dB}$ & $0.25 \mathrm{~dB}$ \\
$\begin{array}{l}\text { Attenuation time } \\
\text { interval }\end{array}$ & $20 \mathrm{~s}$ & $30 \mathrm{~s}$ & $2 \mathrm{~s}$ \\
\hline
\end{tabular}

Table 2 Characteristics of the jammers used for the tests

\begin{tabular}{llll}
\hline Parameter & $\begin{array}{l}\text { Jammer 1-on } \\
\text { L1 }\end{array}$ & Jammer 2 & $\begin{array}{l}\text { Jammer 1-on } \\
\text { E5b }\end{array}$ \\
\hline Sweep period & $9.1 \mu \mathrm{s}$ & $6.3 \mu \mathrm{s}$ & $9.1 \mu \mathrm{s}$ \\
Sweep range & $36.4 \mathrm{MHz}$ & $20.3 \mathrm{MHz}$ & $45.7 \mathrm{MHz}$ \\
$\begin{array}{l}\text { Center Fre- } \\
\text { quency }\end{array}$ & $1564.32 \mathrm{MHz}$ & $1575.5 \mathrm{MHz}$ & $1214.145 \mathrm{MHz}$ \\
\hline
\end{tabular}

The analysis of the Beidou B1c signals is new and was not considered before. A summary of the parameters adopted for the three tests is provided in Table 1. Two jammers were used for the tests. The first one, which was used for Test 1 and Test E5b, has the shape of a cordless phone, is battery powered and cannot be connected to an external antenna: for this reason, it belongs to Group 3 of the classification proposed by Mitch et al. (2011). It is able to broadcast swept signals on different GNSS frequency bands, including GPS L1, GPS L2 and Galileo E5b. The second jammer is also battery power with a Sub-Miniature version A (SMA) antenna connector. For this reason, it belongs to Group 2 of the jammer classification mentioned above. The characteristics of the signals broadcast by these jammers are summarized in Table 2 .

The power profiles obtained for the three tests are analyzed in Fig. 4 that shows the jamming-to-noise power ratio $(J / N)$ estimated from the samples collected using the USRP front end. While a linear $J / N$ profile was expected, see the corresponding parameters in Table 1 , in all three cases, the front end experiences saturation. In this case, the jamming signal is so powerful to exceed the capabilities of the front-end quantization function that effectively clips the received samples to the minimum and maximum values allowed by $8 / 16$ bits. Front-end saturation introduces significant signal distortions that further impact receiver operations.

For the third test, the jammer attenuation is increased again after $550 \mathrm{~s}$ from the start of the test. The samples 


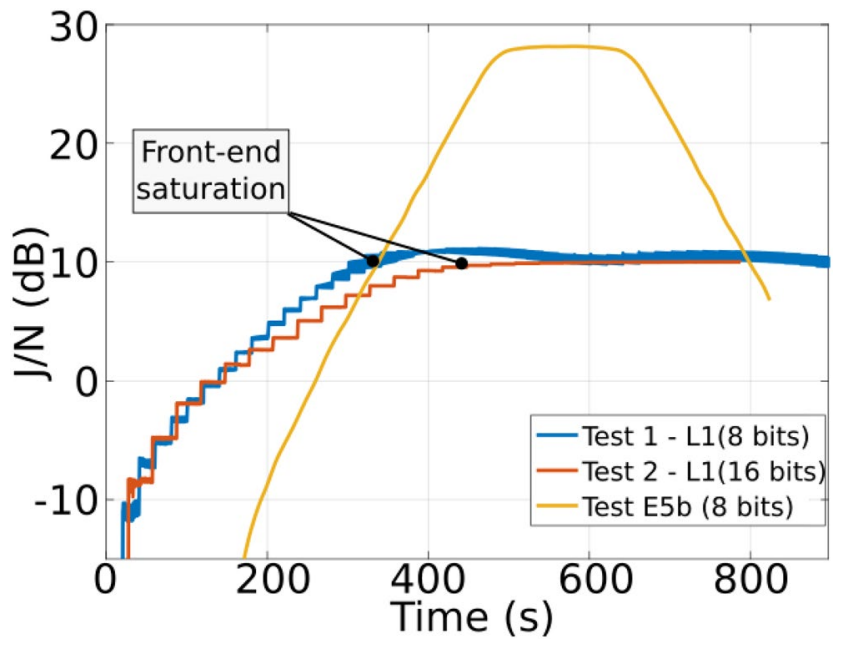

Fig. $4 \mathrm{~J} / \mathrm{N}$ profiles estimated for the three experiments considered in this study. In all cases, front-end saturation occurs

collected using the USRP have been processed either with or without interference mitigation. As a result, several RINEX files with the related GNSS measurements were obtained. The measurements were processed to determine the clock bias and drift solutions analyzed in the next section.

\section{Experimental results}

In this section, experimental results are provided: potential biases and stability degradations in the clock time series obtained when using interference mitigation techniques are analyzed. The impact of interference mitigation on the clock bias is at first discussed along with the effects on the clock drift. In the multiconstellation case, the impact on the intersystem bias is also assessed. Stability analysis is then performed. A total of seven configurations were considered:

- without mitigation;

- interference mitigation using the four RIM techniques described in the section on interference mitigation techniques;

- interference mitigated using the ANF with two pole contraction factor values: 0.8 and 0.9 , respectively.

\section{Clock and intersystem bias analysis}

The impact of interference mitigation techniques on the clock bias time series is analyzed in Fig. 5 for Test 1 . The figure provides the clock bias differences between the baseline solution, without mitigation, and the six interference mitigation techniques presented above. GPS, Galileo and Beidou L1 signals are considered in the top, middle and bottom panels. From the three boxes of the figure, it emerges that RIM techniques do not introduce biases on the clock bias and the differences obtained using these approaches are zero mean. This fact was expected since RIM techniques do not introduce biases on the pseudoranges, as shown by Borio and Gioia (2020a). This result is also confirmed for the Beidou case that was not analyzed before, i.e., no biases are introduced by RIM on the pseudoranges and the clock bias solution.

The time series obtained for the Beidou signal and shown in the bottom part of Fig. 5 further confirm this finding. The time-series variances in Fig. 5 progressively increase with time: this is because the jamming power is progressively increased, as shown in Fig. 4. In the baseline solution, interference is not mitigated, and its effect is visible in the clock bias differences shown in Fig. 5. As already discussed in Borio and Gioia (2020a), FDPB leads to the worst performance and an early loss of lock occurs for the GPS and Galileo cases. Better performance is obtained for the Beidou signals: this is mainly due to the fact that in the Beidou case, the size, $N$, of the FFT used to bring the samples into the frequency domain has been set equal to $T_{c}$. $f_{s}$, where $T_{c}=10 \mathrm{~ms}$ is the code duration of the Beidou B1c signal. Thus, a larger FFT size was used for Beidou signals with respect to Galileo ( $\left.T_{c}=4 \mathrm{~ms}\right)$ and GPS ( $\left.T_{c}=1 \mathrm{~ms}\right)$. This result shows that the FFT size of frequency-domain RIM techniques significantly affects the performance of this type of interference mitigation techniques. The sensitivity analysis of frequency-domain RIM techniques with respect to the FFT size is outside the scope of this work.

Differently from RIM techniques, the ANF introduces biases of several tens of nanoseconds on the clock bias. This result clearly emerges in the three panels of Fig. 5. The biases introduced are modulation dependent and are mainly determined by the position of the frequency notch of the ANF with respect to the main spectral components of the BPSK modulation, used by GPS L1 C/A signals, and of the BOC $(1,1)$ component tracked for the Galileo and Beidou cases. In this respect, the same bias is introduced for the Beidou and Galileo cases.

In order to better understand the impact of the ANF, its behavior has been analyzed at different time instants during the whole duration of Test 1 . Only the case for $k_{\alpha}=0.8$ is detailed in the following: since similar results were obtained for $k_{\alpha}=0.9$, they are not presented to avoid repetitions. Figure 6 provides a characterization of the signals observed at the beginning of Test 1 , after $20 \mathrm{~s}$ from the start of the experiment. The top part shows the Power Spectral Densities (PSDs) of the ANF input and output signals. The ANF transfer function is also provided. This transfer function has been shifted down to about $45 \mathrm{~dB}$ to improve figure clarity. After $20 \mathrm{~s}$ from the start of the test, the $J / N$ is less than $-15 \mathrm{~dB}$, and the impact of jamming can be neglected. 
Fig. 5 Clock bias differences between the baseline solution without mitigation and the six interference mitigation techniques. GPS L1 C/A modulation (top), Galileo E1C signals (middle), and Beidou B1C pilot components (bottom). Test 1
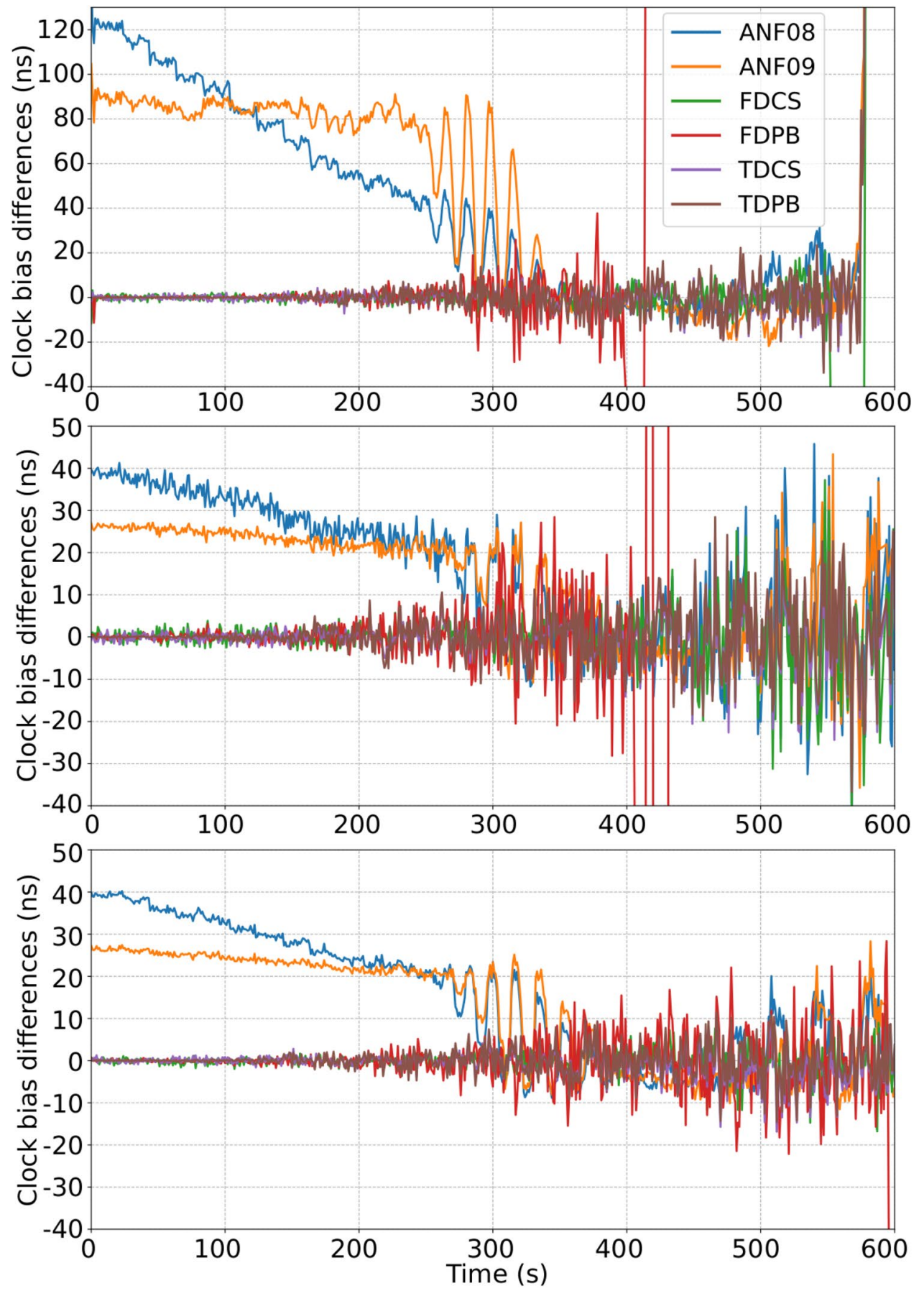

The only interfering term is a $\mathrm{CW}$ generated by the local oscillator of the USRP. The notch of the ANF is quite wide, and the energy at its output is minimized by placing the notch in correspondence of the CW and of the GPS L1 C/A main spectral lobe. For this reason, large portions of the GPS L1 C/A signal spectrum are affected by the ANF and a significant delay (about $120 \mathrm{~ns}$ ) is introduced on the GPS L1 clock bias. The Galileo E1c and the Beidou B1c pilot signals feature a split spectrum, which is less affected by the ANF in the configuration shown in Fig. 6. In this case, delays up to $40 \mathrm{~ns}$ are experienced.

The bottom panel of Fig. 6 shows the spectrogram of the collected samples and the frequency, $f_{0}$, estimated by the ANF. The figure highlights the fact that the notch zero, $z_{0}$, is affected by small oscillations in phase. While not visible in the bottom panel, also the amplitude of $z_{0}$ fluctuates. These fluctuations make the delay introduced by the ANF difficult to predict. 
Fig. 6 PSDs of the signals at the input and output of the ANF $\left(\mathrm{k}_{\alpha}=0.8\right)$. The transfer function of the ANF has been shifted in order to improve clarity (top). Spectrogram of the collected samples and frequency of the ANF notch (red dashed line) (bottom). Test 1, samples were collected after $20 \mathrm{~s}$ from the start of the experiment
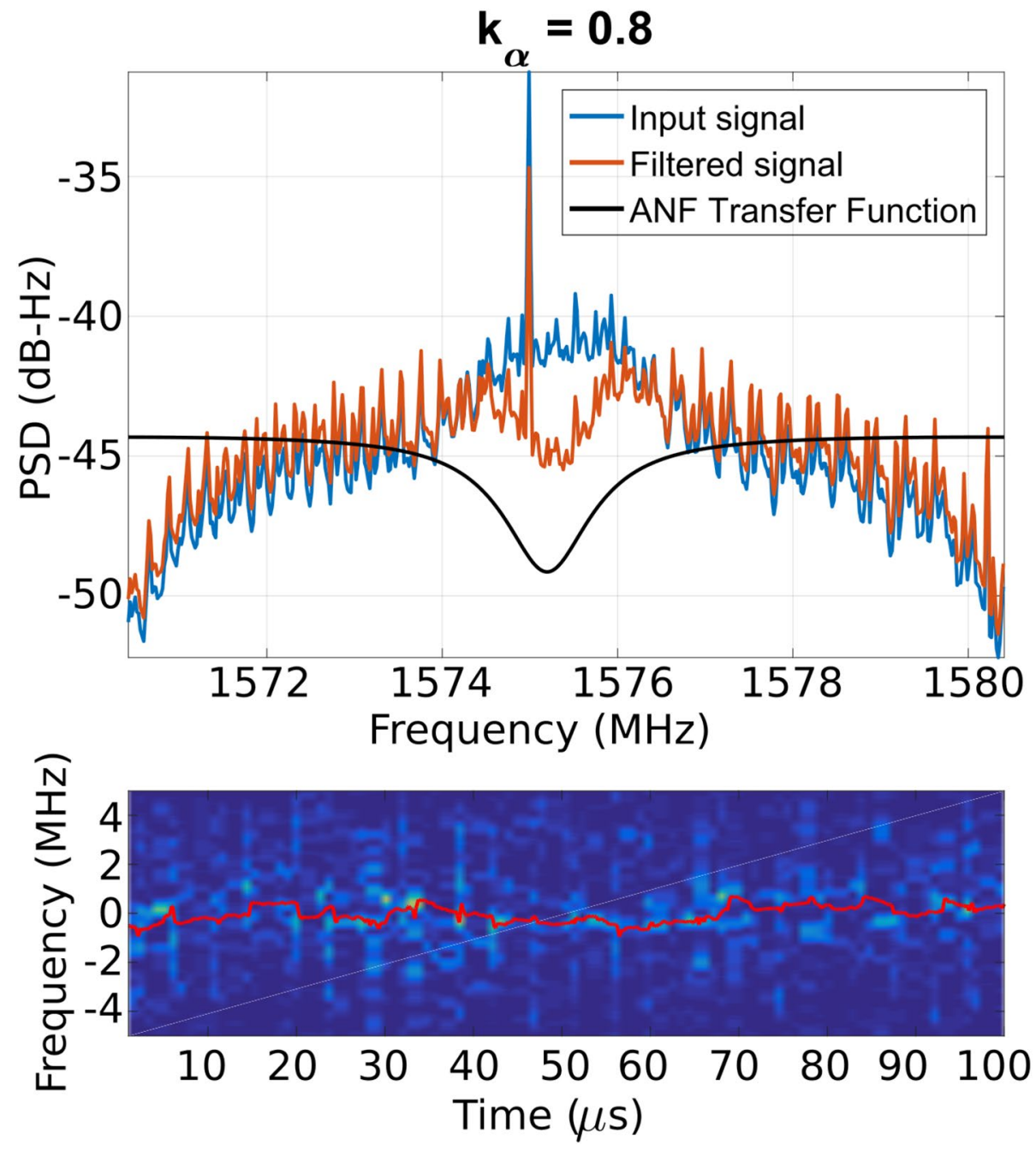

Figure 7 repeats the ANF analysis using samples collected after $420 \mathrm{~s}$ from the start of Test 1 . The PSDs of the signals at the ANF input and output are provided in the top panel along with the transfer function of the ANF. In this case, the collected samples are heavily affected by jamming and significant jamming power is concentrated in the high-frequency region between 1579 and $1580 \mathrm{MHz}$. For this reason, the ANF places its notch around this region. The notch is far from the main spectral components of the three modulations considered in this work. In correspondence of such components, the filter response is practically flat and the delay introduced is negligible. This ANF configuration justifies the reduced delay observed in Fig. 5 for the ANF clock bias differences. The bottom part of Fig. 7 shows the corresponding spectrogram and the frequency estimated by the ANF: reduced frequency variations can be observed with respect to the case analyzed in Fig. 6. The analysis has been repeated at regular intervals for the whole duration of the test, observing different behaviors, including fast variations in the frequency estimated by the ANF.
While, in general, it is difficult to predict the delay introduced by the ANF, it is possible to study its behavior when its zero assumes a constant value. This can be assumed as a first approximation of the cases considered in Figs. 6 and 7. When the zero of the ANF is fixed, the ANF becomes a Linear Time-Invariant (LTI) system and introduces constant delays on the input signals. These delays are a function of the input signal modulation and of the notch center frequency. Thus, the delays can be tabulated and used for correcting the measurements, as discussed in Di Grazia et al. (2019). These corrections can only be applied when the notch zero is fixed.

For the analysis, the value of the zero of the ANF has been recorded at regular time intervals. Then, clean GPS L1 C/A and Galileo E1c signals have been processed with a notch filter with a fixed zero. The delay introduced by these notch filters with fixed zero is then been estimated and compared with the values observed for the ANF in Fig. 8 . The vertical bars represent the delays introduced on the BPSK and BOC $(1,1)$ modulations considering notch filters with a fixed zero. The zeros were extracted from the values 

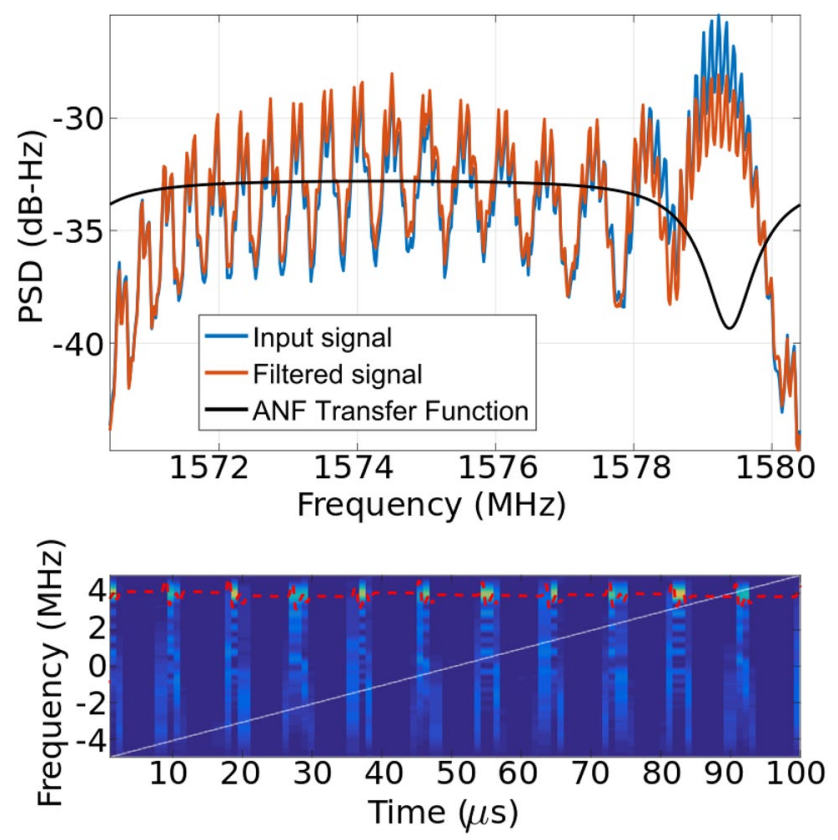

Fig. 7 PSDs of the signals at the input and output of the ANF $\left(\mathrm{k}_{\alpha}=0.8\right)$. The ANF transfer function is also provided (top). Spectrogram of the collected samples and frequency of the ANF notch (red dashed line) (bottom). Test 1, samples collected after $420 \mathrm{~s}$ from the start of the experiment

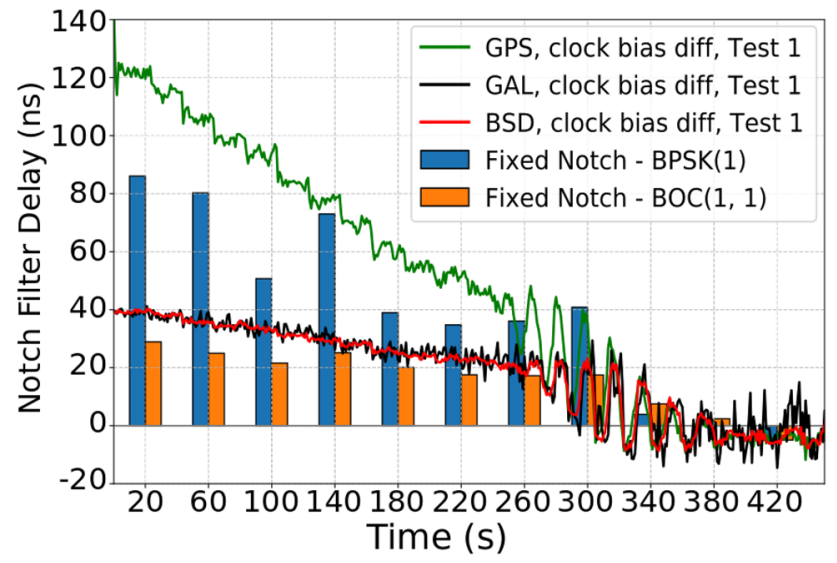

Fig. 8 Analysis of the delay introduced by the ANF. Vertical bars represent delays introduced on the BPSK and BOC modulations considering a notch filter with a fixed zero. Continuous lines are clock bias differences from Fig. 5

estimated by the ANF at the corresponding time instants in Test 1 . The continuous lines in the figure are clock bias differences extracted from Fig. 5. The analysis conducted using nonadaptive notch filters confirms the results obtained in Fig. 5: although a perfect match with the behavior of the ANF cannot be expected, similar trends are observed. Standard notch filters can introduce delays of several tens of nanoseconds. Moreover, the configurations obtained with the ANF for the first part of the test impact more the GPS L1 C/A signal than the Galileo E1c/Beidou B1c component. This is due to the spectral characteristics of the filter transfer function.

The impact of the different mitigation techniques on the intersystem biases is analyzed in Fig. 9 for Test 1. In this case, intersystem bias time series are analyzed separately, and differences are not formed with the standard case, which is also provided in Fig. 9 with the label 'NO MIT'.

It is noted that the time series in Fig. 9 are not the actual GGTO and BGTO, but are the intersystem biases as estimated by the receiver and include hardware and software delays as introduced by the front end and by the processing schemes used to determine the timing solution. No calibration with respect to the actual system time offsets was implemented.

From Fig. 9, it emerges that no bias is introduced by RIM also on these time series. On the contrary, the ANF affects the intersystem bias between systems adopting different signal modulations. Consider, for example, the GPS-Galileo case in the top panel: the intersystem bias obtained when using the ANF with $k_{\alpha}=0.8$ is affected by a difference up to $90 \mathrm{~ns}$ with respect to the standard case. This is because the two systems adopt different modulations that are affected differently by the ANF. The GPSBeidou case is considered in the middle panel. Results similar to those observed for the GPS-Galileo case are observed. When the Galileo-Beidou signals are considered (bottom panel), the intersystem biases are not affected by the ANF, which delays the signals transmitted by the two systems equally. A similar result is expected for the GPS L1C signal (Hein et al. 2006) that will adopt the TimeMultiplexed BOC (TMBOC) modulation and will be interoperable with both Galileo and Beidou signals. Thus, the use of modulations with the same spectral characteristics will avoid biases in the intersystem biases as shown in the bottom panel. These results can be exploited to design efficient positioning techniques that, for example, constrain the time evolution of the intersystem biases as described in Gioia and Borio (2016).

The same analysis conducted for Test 1 and discussed above has been repeated for Test 2 . Since similar findings were obtained, only the clock bias differences between the standard solution and the six interference mitigation techniques are provided in Fig. 10. As for the previous test, GPS, Galileo and Beidou L1 signals are considered in the top, middle and bottom parts of the figure, respectively. In all three cases, the time series provided confirm the findings obtained for Test 1: RIM techniques do not introduce biases, whereas the ANF introduces modulation-dependent delays. In this respect, no significant differences were found when moving from eight to 16 bits. Results similar to those shown in Fig. 9 were found for the intersystem biases: also, in this 
Fig. 9 Intersystem biases for the different interference mitigation techniques. GPS-Galileo (top), GPS-Beidou (middle) and Galileo-Beidou (bottom), Test 1
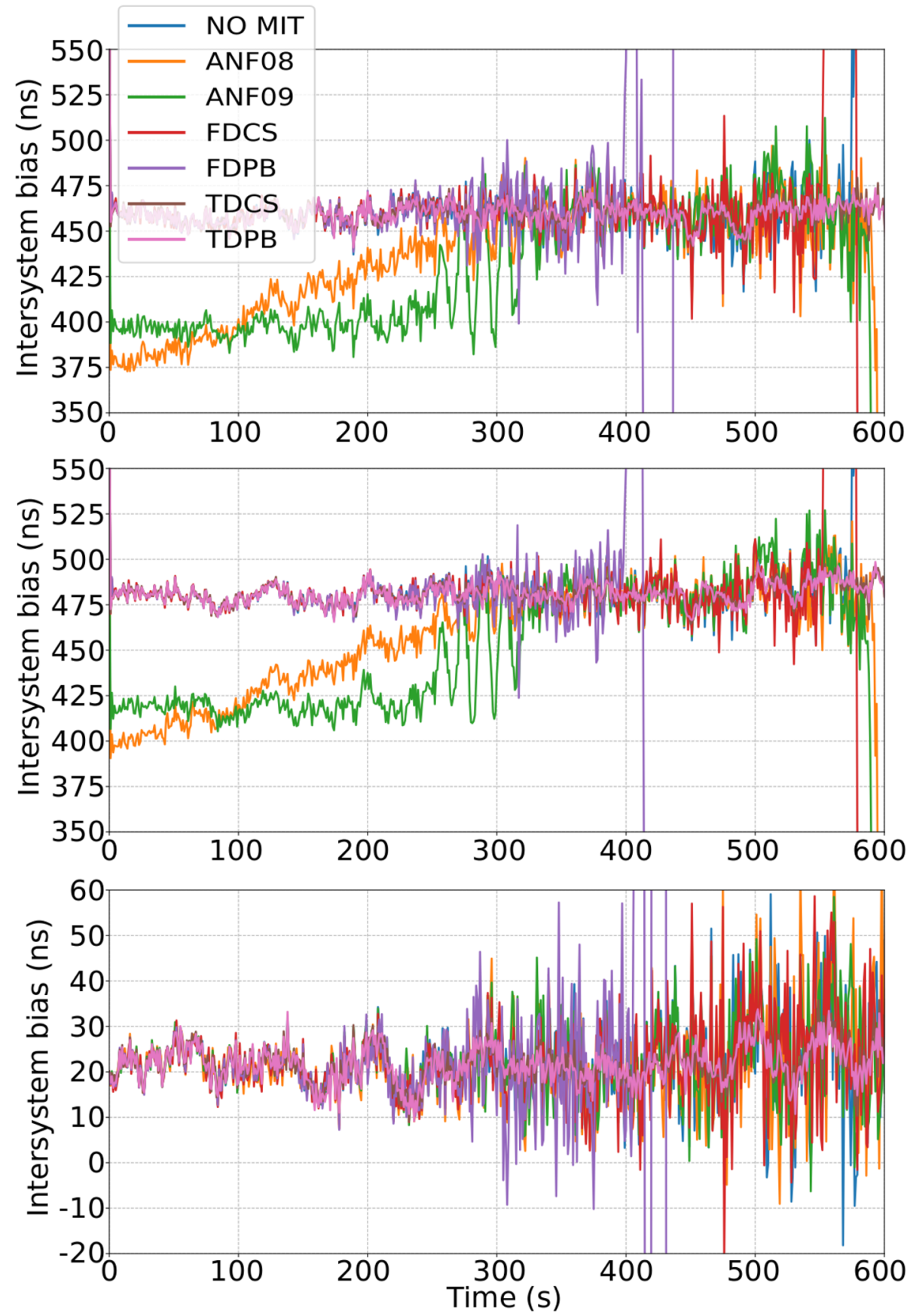

case, the ANF does not affect systems adopting modulations with the same spectral characteristics.

Results for the Galileo E5b signal are finally provided in Fig. 11. As for the Galileo E1c and Beidou B1c signal, the pilot component (Quadrature signal) was used for the measurement generation allowing the use of a pure PLL. Also, in this case, the clock bias differences are zero mean for RIM techniques, whereas time-varying biases can be observed for the ANF with $k_{\alpha}=0.8$ and $k_{\alpha}=0.9$. In the first part of the test shown in the figure ([0-300] s interval), the impact of jamming can be neglected. In this part of the test, the clock bias differences observed for RIM techniques are in the order of a few picoseconds and are significantly lower than the values observed in Figs. 5 and 10. Also, in the ANF cases, the delays observed are significantly lower than those observed in Test 1 and 2. These reduced differences are due to the wideband nature of the Galileo E5b signal for which maximum delays in the order of $3 \mathrm{~ns}$ are observed for the ANF with $k_{\alpha}=0.8$. 
Fig. 10 Clock bias differences between the standard solution without mitigation and the six interference mitigation techniques. GPS L1 C/A modulation (top), Galileo E1c signals (middle) and Beidou B1c pilot components (bottom), Test 2
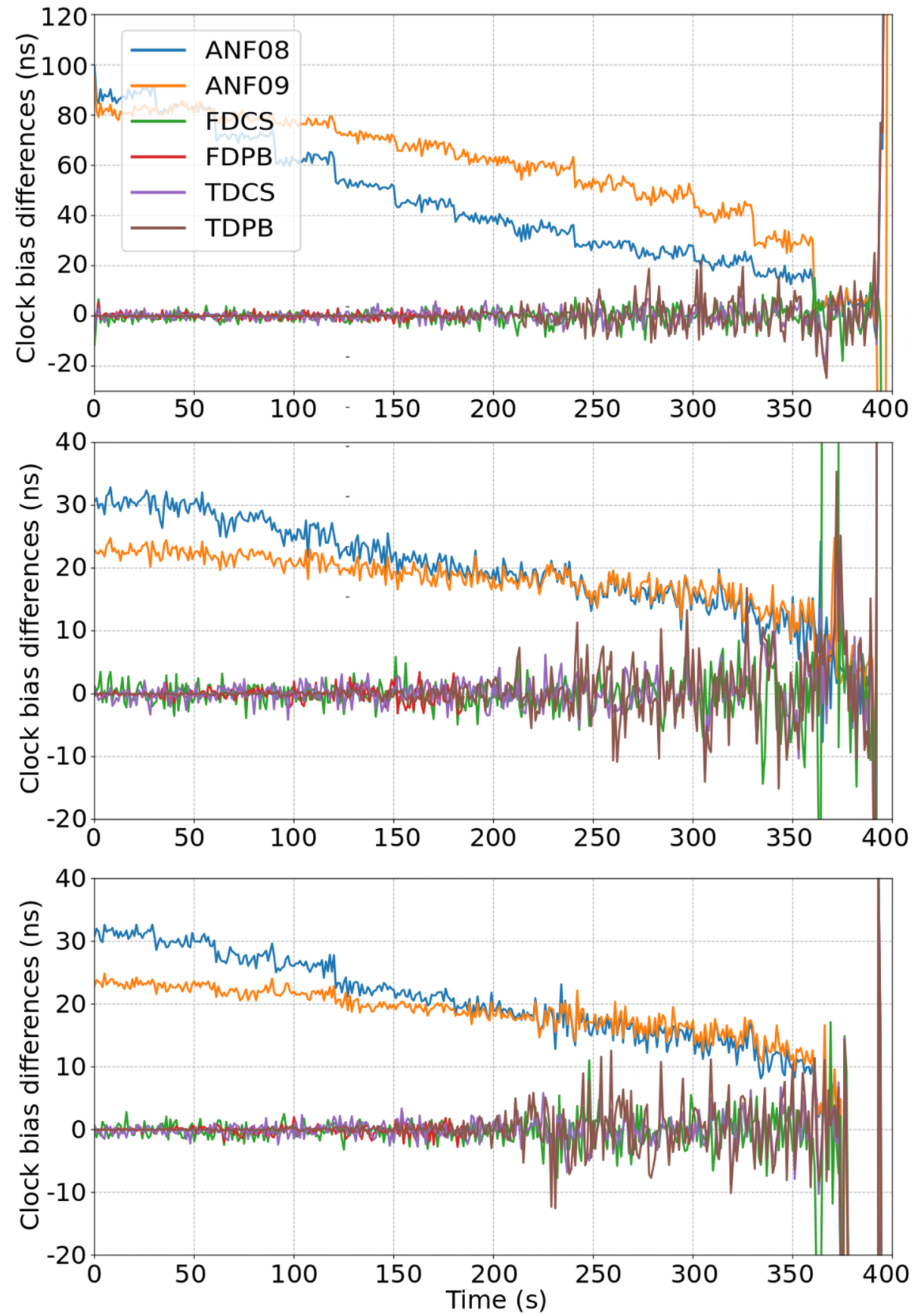

\section{Clock drift analysis}

Clock drift differences caused by interference mitigation techniques were also analyzed. Figure 12 shows sample results showing the clock drift differences obtained for Test 1. As for the previous analysis, GPS L1 C/A signals are considered in the upper part of the figure, Galileo E1c components in the middle panel and Beidou B1c pilot modulations in the bottom panel. In this case, neither biases nor time-varying patterns can be observed. All the interference mitigation techniques considered, including the ANF, do not introduce frequency offsets, and clock drift differences, computed with respect to the case without mitigation, are zero mean. As for the clock bias case, the variance of the clock drift difference increases with time, reflecting the fact that the jamming power is progressively increased. During the first $200 \mathrm{~s}$ of Test 1 , the $J / N$ is less than $5 \mathrm{~dB}$ and the impact of jamming can be neglected. In this part of the test, the clock drift differences are less than $10 \mathrm{ps} / \mathrm{s}$ in magnitude for the three 
Fig. 11 Clock bias differences between the standard solution without mitigation and the six interference mitigation techniques. Test E5B, Galileo E5b pilot signal

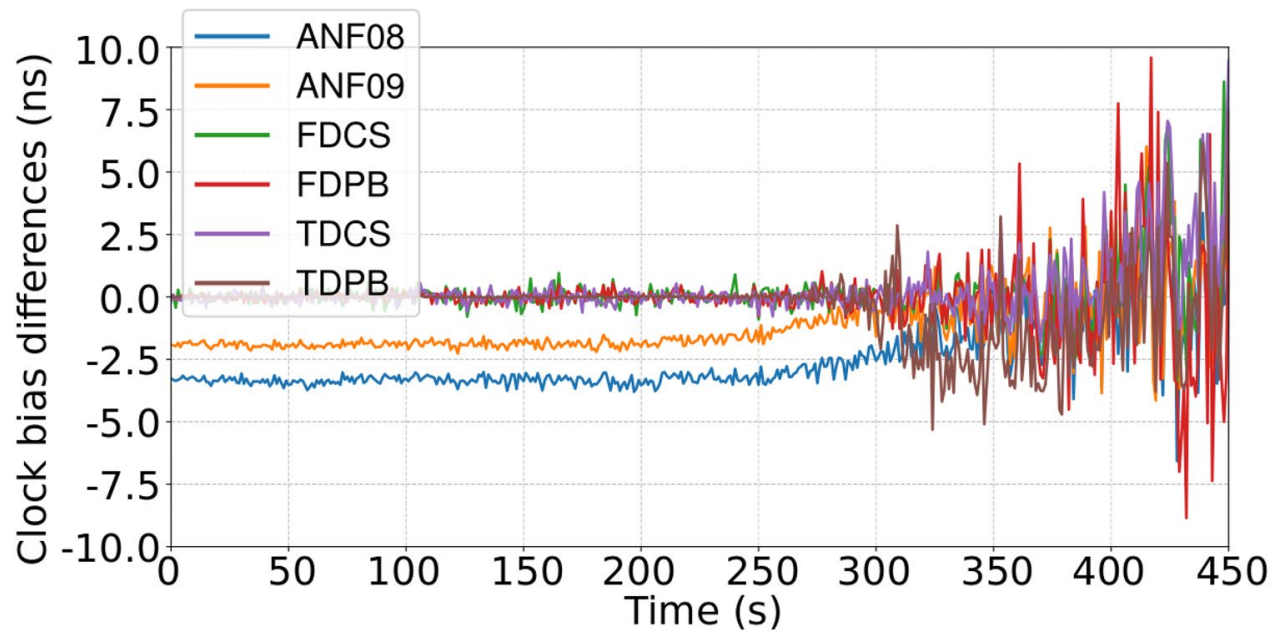

modulations considered. These values are within the residual processing noise present in the clock drift estimates.

Similar results were obtained for Test 2 and Test E5b. These findings, which are not repeated here, confirm the fact that RIM techniques and the ANF do not bias the clock drift estimated by the receiver for the different GNSS modulations.

\section{Stability analysis}

In this section, results in terms of OADEV are provided. The OADEV is an efficient estimator for the ADEV (Riley 2008; Bregni 2002), and it is used to assess the effects of interference mitigation on the short-term stability of the clock bias and drift solutions estimated using different types of GNSS signals and in the presence of jamming. In the following, the terms ADEV and OADEV are used interchangeably.

This analysis aims to assess the impact of interference mitigation on the short-term stability of the clock solutions. It is noted that the clock bias and drift estimated using the approach discussed above characterize the behavior of the front-end local clock: in this case the clock of a USRP II. In this respect, stabilities in the $10^{-9}$ order are obtained. These stabilities do not characterize the final performance of a timing receiver, which provides a timing output steered to a GNSS time scale, but characterize the clock bias and drift estimated by the receiver. The analysis is performed to verify that interference mitigation does not degrade the stability of the clock bias and drift. For large time intervals, all ADEV curves should converge and characterize the medium-term behavior of the USRP II clock. At short term, two contributions should be present: the intrinsic short-term noise of the USRP II clock and the additional processing noise introduced by the different signal processing stages used to estimate the clock bias and drift time series. While the local clock's intrinsic short term noise is not affected by interference mitigation, the processing noise contribution may change depending on the adopted interference mitigation technique. An increase in the ADEV at short averaging intervals would indicate a performance degradation caused by an interference mitigation technique.

In the following, portions of the tests where the impact of jamming is relatively low and all the techniques lead to valid timing solutions are considered. The ADEV curves for the different processing approaches analyzed for Test 1 are shown in Fig. 13, which considers the GPS L1 C/A case. In the upper part of Fig. 13, ADEVs are computed using the clock bias time series in the [0-400] time interval. As expected, for averaging intervals greater than $20 \mathrm{~s}$, all curves converge, describing the medium-term stability behavior of the USRP clock. In the short term, only limited variations can be observed. In the baseline case without mitigation, a stability equal to $9 \times 10^{-9}$ is observed for $\tau=1 \mathrm{~s}$. This result partially takes into account the effect of jamming that starts influencing the receiver performance in the portion of the test between [250-400] s.

Time-domain RIM processing improves receiver performance, and an ADEV equal $5 \times 10^{-9}$ is obtained for $1 \mathrm{~s}$ averaging time. The other techniques achieve performance close to that achieved by the baseline approach. In agreement with the results obtained in (Borio and Gioia 2020a), FDPB provides the worst performance and a slight degradation in ADEV is observed. However, the degradation is limited $\left(A D E V(1)=1.3 \cdot 10^{-8}\right)$ and confirms that RIM introduces a limited noise increase in the absence of interference.

In the bottom part of Fig. 13, ADEV curves have been computed using the clock drift time series. In this case, practically all the curves coincide and only FDPB introduces some short-term stability degradations. In the medium/long term, the same behavior obtained using clock biases is found. The analysis has been repeated using Galileo and Beidou signals. Since the two cases led to 
Fig. 12 Clock drift differences between the standard solution without mitigation and the six interference mitigation techniques. GPS L1 C/A modulation (top), Galileo E1c signals (middle) and Beidou B1c pilot components (bottom), Test 1
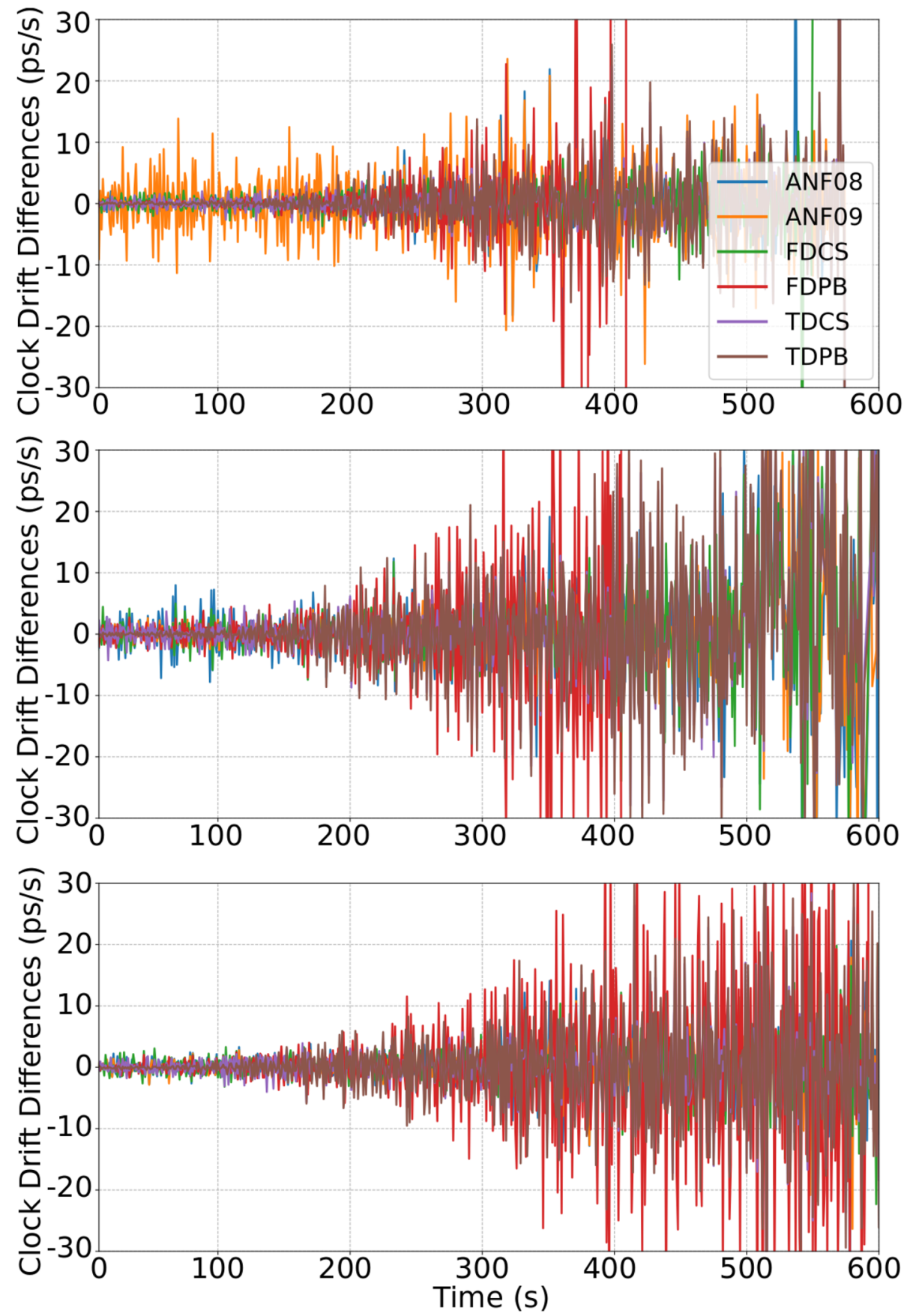

similar results, only Galileo processing is considered as shown in Fig. 14. As for the GPS case, all the time series led to the same medium-term behavior. Moreover, results similar to those obtained in Fig. 13 are found. This fact was expected since, in both cases, the local oscillator stability is analyzed and the GPS and Galileo time scales are used as reference. Differences between GNSS time scales are several orders of magnitude lower than the local oscillator instabilities, and no noticeable differences should be present in the ADEV curves. In the top panel, a short term behavior similar to that observed for the GPS case is found where only limited variations can be appreciated in the ADEV curves. In the bottom panel, no differences can be appreciated among the curves obtained using the different techniques. In this case, the ADEV curves were computed using clock drift time series. This result further supports the fact that interference mitigation techniques only marginally affect GNSS timing receiver's performance in the absence of jamming or for low levels of interference. Results obtained for Test 2 are omitted to avoid the repetition of similar findings. 

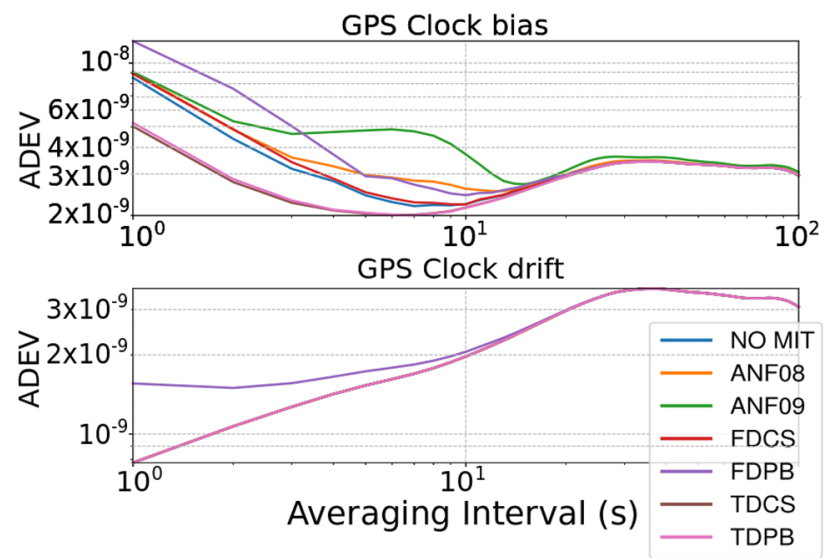

Fig. 13 ADEV curves for the different processing approaches. ADEV from the clock bias (top) and from the clock drift (bottom). Measurements in the [0-400] s time interval of Test 1,8 bit. GPS L1 C/A case
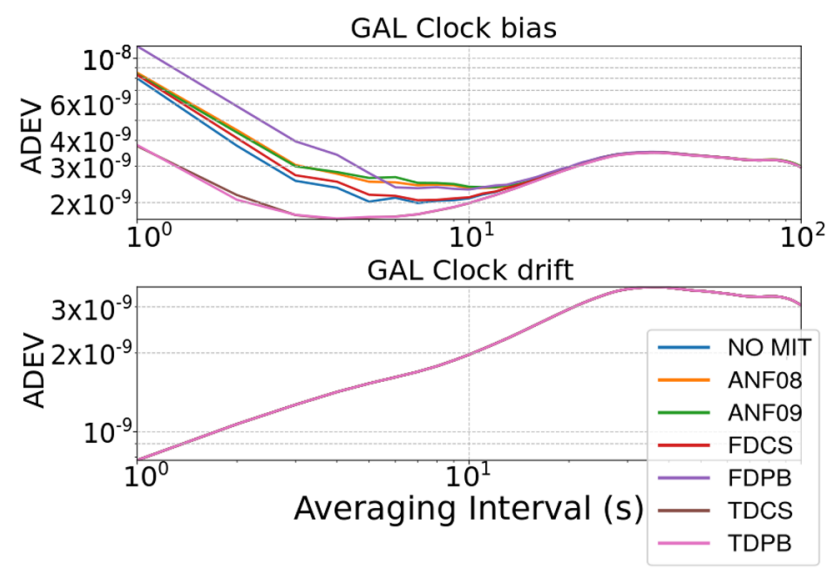

Fig. 14 ADEV curves for the different processing approaches. ADEV from the clock bias (top) and from the clock drift (bottom). Measurements in the [0-400] s time interval of Test 1,8 bit. Galileo E1c case

Finally, ADEV results obtained for Test E5b are shown in Fig. 15. The ADEV has been computed using clock bias (top panel) and drift values (bottom panel) obtained during the first $400 \mathrm{~s}$ of the experiment. In this time interval, the receiver is able to maintain signal lock and provide a clock solution for all the seven techniques analyzed. From the figure, it emerges that for the Galileo E5b experiment, differences in the short term are even lower than in the previous cases. In the clock bias case analyzed in the top panel, only negligible differences can be observed for an averaging
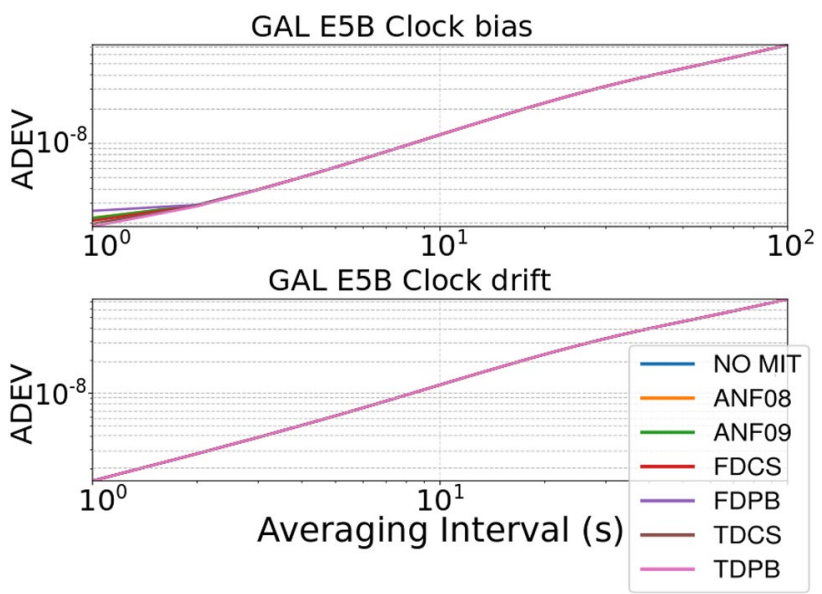

Fig. 15 ADEV curves for the different processing approaches. ADEV from the clock bias (top) and from the clock drift (bottom). Measurements in the [0-400] s time interval of Test E5b, 8 bit. Galileo E5b signal

interval, $\tau$, equal to $1 \mathrm{~s}$. When the ADEV is computed from the clock drift (bottom panel), all the curves coincide.

Also, for the Galileo E5b experiment, the ADEV results show a limited impact in terms of noise increase caused by interference mitigation. This result is valid for low levels of interference, which are considered by limiting the analysis to the first $400 \mathrm{~s}$ of the experiment.

\section{Conclusions}

We studied the impact of interference mitigation on the timing solution of a GNSS timing receiver. Five precorrelation interference mitigation techniques were analyzed, including four RIM approaches and the ANF. For the analysis, different tests and different GNSS modulations were considered. Experiments were performed on the L1 and $\mathrm{E} 5 \mathrm{~b}$ frequencies and included a multiconstellation configuration with GPS L1 C/A, Galileo E1b/c and Beidou $\mathrm{B} 1 \mathrm{c}$ signals. The analysis focused on the timing solution obtained by fixing the user position and velocity. In this way, the impact of interference mitigation on the clock bias, clock drift and intersystem biases was assessed.

This work complements previous results in the position domain and shows that RIM techniques, when properly configured, do not degrade receiver performance in terms of time retrieval. The analysis shows that RIM does not introduce biases in the clock time series. Moreover, it can 
lead to a reduction in the short-term noise as quantified by the ADEV.

On the contrary, the ANF introduces a time-varying delay on the clock biases. This delay is modulationdependent and is difficult to predict. The ANF introduces the same delay on the Galileo E1b/c and Beidou B1c signals: this is because the two signals have the same spectral characteristics. The intersystem bias analysis confirmed that the ANF introduces delays only on intersystem biases of those GNSSs that use modulations with different spectral characteristics. The use of interoperable signals prevents the ANF from introducing additional intersystem delays, which enables advanced processing when intersystem biases are considered practically constant.

From the analysis of the clock drifts, it emerged that all the interference mitigation techniques have a limited impact on these components. No biases were found in the clock drifts determined using interference mitigation techniques. Finally, the ADEV analysis confirmed the limited impact of interference mitigation on the short-term stability of the clock time series.

Open Access This article is licensed under a Creative Commons Attribution 4.0 International License, which permits use, sharing, adaptation, distribution and reproduction in any medium or format, as long as you give appropriate credit to the original author(s) and the source, provide a link to the Creative Commons licence, and indicate if changes were made. The images or other third party material in this article are included in the article's Creative Commons licence, unless indicated otherwise in a credit line to the material. If material is not included in the article's Creative Commons licence and your intended use is not permitted by statutory regulation or exceeds the permitted use, you will need to obtain permission directly from the copyright holder. To view a copy of this licence, visit http://creativecommons.org/licenses/by/4.0/.

\section{References}

Bastide F, Chatre E, Macabiau C, Roturier B (2004) GPS L5 and GALILEO E5a/E5b signal-to-noise density ratio degradation due to DME/TACAN signals: Simulations and theoretical derivation. In: Proceedings of ION NTM 2004, Institute of Navigation, San Diego, California, USA, January 26-28, pp 1049-1062.

Borio D (2017). Robust signal processing for GNSS. In: Proceedings of ENC 2017, Lousanne, Switzerland, May 9-12, pp 150-158.

Borio D, Camoriano L, Lo Presti L (2008) Two-pole and multipole notch filters: a computationally effective solution for GNSS interference detection and mitigation. IEEE Syst J 2(1):38-47

Borio D, Camoriano L, Mulassano P (2006) Analysis of the onepole notch filter for interference mitigation: Wiener solution and loss estimations. In: Proceedings of ION GNSS 2006, Institute of Navigation, Fort Worth, Texas, USA, September 26-29, pp $1849-1860$

Borio D, Closas P (2018) Complex signum non-linearity for robust GNSS signal processing. IET Radar Sonar Navigation 12(8):900-909

Borio D, Closas P (2019) Robust transform domain signal processing for GNSS. Navigation 66(2):305-323

Borio D, Gioia C (2020a) GNSS interference mitigation: a measurement and position domain assessment. Navigation, pp 1-22. Early View: https://doi.org/10.1002/navi.391

Borio D, Gioia C (2020b) Impact of robust interference mitigation on GNSS timing. In: Proceedings of ENC 2020, Dresden, Germany, November 23-24, pp 1-14.

Bregni S (2002) Synchronization of digital telecommunications networks. Wiley, New York

Calmettes V, Pradeilles F, Bousquet M (2001) Study and comparison of interference mitigation techniques for GPS receiver. In: Proceedings of ION GPS 2001, Salt Lake City, Utah, USA September 11-14, pp 957-968.

Di Grazia, D., Cardineau, D., Pisoni, F. (2019). A NAVIC enabled hardware receiver for the Indian mass market. In: Proceedings of ION GNSS+ 2019, Institute of Navigation, Miami, Florida, USA, September 16-20, 189-199.

Dovis F (ed) (2015) GNSS interference threats and countermeasures. Artech House, Norwood, Massachusetts

European Union (2016) European GNSS (Galileo) open service signalin-space control document. Issue 1.3.

Gao GX, Heng L, Hornbostel A, Denks H, Meurer M, Walter T, Enge P (2013) DME/TACAN interference mitigation for GNSS: algorithms and flight test results. GPS Solut 17:561-573

Gao GX, Sgammini M, Lu M, Kubo N (2016) Protecting GNSS receivers from jamming and interference. Proc IEEE 104(6):1327-1338

Gioia C, Borio D (2016) A statistical characterization of the Galileoto-GPS inter-system bias. J Geodesy 90(11):1279-1291

Guo W, Song W, Niu X, Lou Y, Shougang Zhang SG, Shi C (2019) Foundation and performance evaluation of real-time GNSS highprecision one-way timing system. GPS Solut 23:1-11

Hein GW et al (2006) MBOC: The new optimized spreading modulation recommended for Galileo L1 OS and GPS L1C. In: Proceedings of IEEE/ION PLANS 2006, San Diego, California, USA, April 25-27, pp 883-892.

Huber PJ (1964) Robust estimation of a location parameter. Ann Math Statist 35(1):73-101

Huber PJ, Ronchetti EM (2009) Robust statistics, 2nd edn. Wiley Probability and Statistics. John Wiley and Sons, New York

Kaplan ED, Hegarty C (eds) (2005) Understanding GPS: principles and applications, 2nd edn. Artech House Publishers, Norwood

Kuusniemi H (2005) User-level reliability and quality monitoring in satellite-based personal navigation. $\mathrm{PhD}$ thesis, Tampere University of Technology, Finland.

Madhani PH, Axelrad P, Krumvieda K, Thomas J (2003) Application of successive interference cancellation to the GPS pseudolite near-far problem. IEEE Trans Aerospace Electronic Syst 39(2):481-488

Mingquan L, Wenyi L, Zheng Y, Xiaowei C (2019) Overview of BDS III new signals. Navigation 66(1):19-35

Mitch RH, Dougherty RC, Psiaki ML, Powell SP, O'Hanlon BW, Bhatti JA, Humphreys TE (2011) Signal characteristics of civil GPS jammers. ION GNSS 2011, Institute of Navigation, Portland, Oregon, USA, September 20-23, pp 1907-1919. 
Parkinson B, Spilker J, Axelrad P, Enge P (1996) Global positioning system: theory and applications, vol I. C., USA, American Institute of Aeronautics and Astronautics, Washington DC

Qin W, Dovis F, Troglia Gamba M, Falletti E (2019a) A comparison of optimized mitigation techniques for sweptfrequency jammers. In: Proceedings of ION ITM 2019, Institute of Navigation, Reston, Virginia, USA, January 28-31, pp 233-247.

Qin W, Troglia Gamba M, Falletti E, Dovis F (2019b) Effects of optimized mitigation techniques for swept-frequency jammers on tracking loops. In: Proceedings of ION GNSS+ 2019, Institute of Navigation, Miami, Florida, USA, September 16-20, pp 3275-3284.

Raasakka J, Orejas M (2014) Analysis of notch filtering methods for narrowband interference mitigation. In: Proceedings of IEEE/ ION PLANS 2014, Monterey, California, USA, May 5-8, pp $1282-1292$.

Riley WJ (2008) Handbook of frequency stability analysis. Number 1065. NIST Pub Series: Special Publication (NIST SP), Boulder, CO 80305.

Tsui JB-Y (2004). Fundamentals of global positioning system receivers, A Software Approach, 2nd edn. Wiley-Interscience, New York.

Xiangwei Z, Yiwei W, Hang G, Wenxiang L, Feixue W (2015) GNSS timing receiver toughen technique in complicated jamming environments. J Natl Univ Defense Technol 37(3):1-9

Young JA, Lehnert JS (1998) Analysis of DFT-based frequency excision algorithms for direct-sequence spread-spectrum communications. IEEE Trans Commun 46(8):1076-1087

Publisher's Note Springer Nature remains neutral with regard to jurisdictional claims in published maps and institutional affiliations.

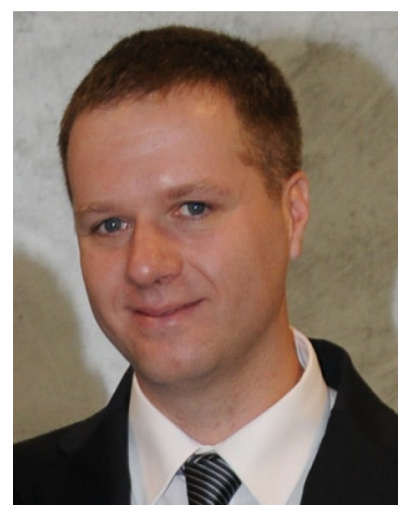

Daniele Borio received the M.S. and the Ph.D. degrees from Politecnico di Torino in 2004 and 2008, respectively. From January 2008 to September 2010, he was a senior research associate in the PLAN group of the University of Calgary, Canada. He is currently a scientific policy officer at the Joint Research Centre of the European Commission in digital and wireless communications, location and navigation.

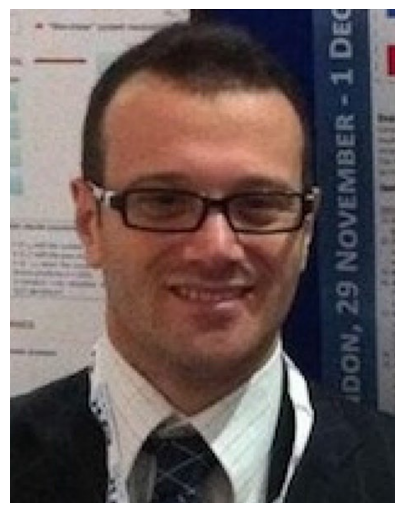

Ciro Gioia received the M.S. in nautical sciences and a Ph.D. degree in geomatics from Parthenope University in 2009 and 2014, respectively. From May 2013 to April 2014, he was a visiting student at the European Commission Joint Research Centre (JRC). From May 2014 to July 2016, he was an external consultant at JRC. Currently, he is scientific project officer at the JRC. His research interest focuses on location and navigation with special emphasis on geomatics aspects. 